\title{
A Case Report on Congenital Adrenal Hyperplasia, Varicella Zoster Infection, Varicella Encephalopathy \& Cerebellitis
}

\author{
Aswathy M Shaji ${ }^{1}$, A. Priya ${ }^{2}$, S Suwitha $^{3}$, K. Arun Chander Yadav ${ }^{4}$ \\ ${ }^{1,3}$ Clinical Pharmacist Apollo Children's Hospital, Chennai, Tamil Nadu, India. \\ ${ }^{2}$ Junior Executive Apollo Children's Hospital, Chennai, Tamil Nadu, India. \\ ${ }^{4}$ Consultant Clinical Pharmacologist Apollo Children's Hospital, Chennai, Tamil Nadu, India.
}

Corresponding Author: K. Arun Chander Yadav

\begin{abstract}
Congenital adrenal hyperplasia (CAH) comprises a family of autosomal recessive disorder and it will disrupt adrenal steroidogenesis. The most common form of $\mathrm{CAH}$ is due to 21-hydroxylase deficiency associated with mutations in the cyp2la2 gene which is located at chromosome 6p21. The clinical features associated with this adrenal steroidogenesis represent a clinical spectrum reflecting to the consequences of the specific mutations. Treatment goals include normal linear growth velocity and "on-time" puberty in affected children. ${ }^{[1}$

Infection with Varicella zoster virus (vzv) causes chickenpox means Varicella that can be severe in immunocompromised individuals, infants and adults. The primary infection is followed by latency in ganglionic neurons. During this time, no virus particles will produce and no obvious neuronal damage occurs. Reactivation of virus leads to virus replication, which will causes zoster (shingles) in tissues innervated by the involved neurons, inflammation and cell death ${ }^{[2]}$. Potential complications of this infection are involved in the central nervous system causing encephalitis. An increased risk of this complication is associated with the immunocompromised patient. ${ }^{[3]}$
\end{abstract}

Keywords: congenital adrenal hyperplasia, varicella zoster infection, varicella encephalopathy, cerebellitis

\section{INTRODUCTION}

Congenital adrenal hyperplasia $(\mathrm{CAH})$ is a group of hereditary disorders and it can affect the adrenal glands. Adrenal glands produce cortisol and aldosterone hormones. Congenital adrenal hyperplasia is caused by genetic defects that prevent these glands from producing these two hormones either entirely or at normal rates. There are two types of $\mathrm{CAH}$, that is classic $\mathrm{CAH}$ and non classic $\mathrm{CAH}$. Classic congenital adrenal hyperplasia diagnosed during infancy, is the more severe of the two types. Children with this condition don't make enough cortisol; these children also produce excess amounts of male sex hormones called androgens. Boys may have enlarged penis, and girls may have ambiguous or masculine-looking genitals. Nonclassic congenital adrenal hyperplasia is more common than classic form. It is diagnosed usually in early adolescence or in younger children who have early signs of puberty. Children with nonclassic CAH have normal levels of aldosterone and excess amounts of androgens. Symptoms often far milder than those associated with classic condition, and many children don't need treatment. The nonclassic form will not progress to the classic form. CAH results in deficiency of any one of these enzymes. Impaired cortisol synthesis may leads to chronic elevations of Adrenocorticotropic hormone (ACTH) via 
the negative feedback system, causing overstimulation of the adrenal cortex and resulting in hyperplasia and over secretion of the precursors to the enzymatic defect. The diagnosis is hormonal diagnosis, which means each form of $\mathrm{CAH}$ has its own unique hormonal profile, consisting of elevated levels of precursors and elevated or diminished levels of adrenal steroid products. Traditionally, laboratories measured urinary excretion of adrenal hormones or their urinary metabolites .However, collection of 24 hour urine excretion is difficult, particularly in neonates. Therefore, the simple and reliable radioimmunoassays are utilized now for measuring circulating serum levels of adrenal steroids. A non-invasive random urine collection in the first days for steroid hormone metabolites and precursor/product ratio assessments can be measured simultaneously. Other diagnoses are prenatal diagnosis of 21ohd, non-invasive prenatal diagnosis of $\mathrm{CAH}$ and preimplantation diagnosis.

The requirement of hydrocortisone (or its equivalent) for the treatment of classical 21ohd form of CAH is about 10-15 $\mathrm{mg} / \mathrm{m} 2 /$ day divided into 2 or 3 doses per day. Dosage requirements for patients with non classic -21ohd $\mathrm{CAH}$ are typically less. Adults be treated with the longer-acting dexamethasone or prednisone, alone or in combination with hydrocortisone. A small dose of dexamethasone will be given at bedtime ( 0.25 to $0.5 \mathrm{mg}$ ) is usually adequate for androgen suppression in non-classical patients

Varicella-zoster virus (vzv) is a pervasive human alpha herpes virus that causes Varicella (chicken pox) and herpes zoster (shingles). Varicella is a common childhood illness, that is characterized by fever, viremia, and scattered vesicular lesions in the skin ${ }^{[4]}$. Varicella is the primary manifestation of a Varicella zoster virus (vzv) infection, is generally mild; indeed, severe complications are seldom reported in immunocompetent children. Nevertheless, hospitalizations due to
Varicella do occur in otherwise healthy children, thereby producing an economic burden on the health care system ${ }^{[5]}$.

The primary infection with the virus causes varicella. A decline in the cellmediated immunity results in virus reactivation causing a spectrum of neurological syndromes such as herpes zoster, vasculopathy, myelopathy, retinal necrosis, cerebellitis, and zoster sine herpete. Primary Varicella infection predominantly affects children $<10$ years of age. ${ }^{[6]}$

Among neurological complications, acute cerebellitis (ac) was the most frequent manifestation. However, the occurrence of ac complicating Varicella in hospitalized children and its detailed clinical characteristics are unknown ${ }^{[7]}$.

\section{CASE REPORT}

5 years 10 months old male child came to hospital with known case of congenital adrenal hyperplasia at the age of 1 month following which child was started on Tab. Hisone and Tab. Fludrocort. He came with the complaints of fever with rashes since 5 days, fever since 2 days high grade intermittent fever associated with chills and rigors. Vesicular rashes initially formed in the trunk and spread to face and gradually ruptured for which he was treated with oral azithromycin. History of GTCS, upward gaze, History of altered sensorium irritability ( 2 days) followed by history unsteadiness of gait. No history of focal deficits/ weakness of limbs/ cranial nerve deficits. No history of contact with Varicella, travel history. MRI done- normal. He was referred from other hospital with iv hydrocort stress dose advised ivig and parents sought further care at Apollo and he was treated with iv acyclovir. During admission time the general appearance is active vesticles present scabs present all over the body and generalized hyper pigmentation of skin. Child brought with above mentioned complaints, on arrival afebrile and hemodynamically stable. CBC. $\mathrm{Rp}$-ii, urine routine, COVID RTPCR done. 
CBC showed mild hemoglobin low level, leucopenia, with neutropenia, mild thrombocytopenia seen. Urine routine RT-ii and SGPT were normal. He was treated with inj. Acyclovir, inj. Ceftriaxone, Inj. Levipil, tab. Hisone was increased to double strength dose due to acute infective condition. Id opinion was obtained and advised to continue inj. Acyclovir and stop Ceftriaxone and no need for ivig infusion/ CSF analysis since child improving better and infection on resolving condition. Neurologist opinion was obtained and advised to continue inj. Acyclovir and no need for CSF analysis/ ivig infusion for now since infection in resolving condition. Endocrinologist opinion: child was continued on stress dose of steroids, the electrolytes and bp were normal. Stretched penile length was longer for age while testicular volume was prepubertal, skin and genitalia were hyper pigmented, the child has peripheral precocious puberty probably due to poor compliance to medication. During discharge he was hemodynamically stable.

\section{DISCUSSION}

Acute cerebellitis is the most common neurological complication of Varicella, occurs about once in 4000 Varicella cases among children. Nevertheless, it has been scarcely studied and it is still debated whether it has a post infectious, immunologic pathogenesis or a primarily infectious origin ${ }^{[7]}$. 5 year 10months old child developmentally had a complaints of seizure in the form of uprolling of eyes and tonic clonic movements of bilateral upper limbs and child diagnosed as a case of congenital adrenal hyperplasia which was started on tab hison and fludrocort on examination he have vesicles over trunk and face, and generalized hyper pigmentation and from neurologist opinion child was advised to take the MRI and there is no significant abnormalities. Neurologist reviewed the child and advised to give ivig in view of ataxia, increased irritability. For this he treated on iv hydrocortisone $80 \mathrm{mg} / \mathrm{kg}$ loading followed by $20 \mathrm{mg}$ q6th hourly,iv levipil $40 \mathrm{mg} / \mathrm{kg}$ followed by $20 \mathrm{mg} / \mathrm{kg} /$ day and iv acyclovir $400 \mathrm{mg}$ for 2 days and for some other social reasons child brought apollo hospital, on arrival he was afebrile and hemodynamically stable. He was treated with inj. Acyclovir, inj. Ceftriaxone, inj. Levipil; tab. Hisone was increased to double strength dose due to acute infective condition. The neurological complication of Varicella infection is central nervous system diseases result from reactivation of latent infection in the immunosuppressive conditions such as old age, diabetes, cancer, immunosuppressive drugs, and from childhood onwards the patient is used to take Tab. Hisone and Tab. Fludrocort for $\mathrm{CAH}$ and he does not taken the Varicella vaccine. Here the patient is having symptomatic treatment and mostly because of the zoster virus infection child may able to get seizure and cerebellitis. For congenital adrenal hyperplasia child was on oral and iv hisone, followed by inj levipil for seizures. Child was treated on antiviral (acyclovir) and antibiotic (Ceftriaxone) for the Varicella zoster infection. During discharge child was hemodynamically stable and advised to take floricot $100 \mathrm{mcg}$ tab, tab valacyclovir $500 \mathrm{mg}$, pan $20 \mathrm{mg}$ tab, hisone $15 \mathrm{mg}$ tab, levipil syrup $2 \mathrm{ml}$.

The importance of compliance to medication reinforced and advised x-ray left hand with wrist ap view, serum 17ohp level, testosterone, dheas, Lh levels. And on 13april 2021 they came to do the test and results as normal only.

\begin{tabular}{|l|l|}
\hline Test name & Result \\
\hline 17-a-hydroxyprogesterone - serum(Elisa) & $<3.0$ \\
\hline Lh: luteinizing hormone-serum(chemiluminescence) & $<0.1$ \\
\hline Testosterone - serum (electrochemiluminescence:eclia) & $<2.0$ \\
\hline $\begin{array}{l}\text { Dehydroepiandrosterone sulphate (dhea-s) - } \\
\text { serum(chemiluminescence) }\end{array}$ & $<1.0$ \\
\hline
\end{tabular}

\section{CONCLUSION}

The patient was managed 4 days in the hospital and made good progress. He was discharged home with oral hydrocortisone, fludrocortisone acetate, valacyclovir 500mg and levipil syp $2 \mathrm{ml}$. Data from this study may help to better 
address the problem and the treatment of congenital adrenal hyperplasia, Varicella cerebellar complications in hospitalized children.

\section{Acknowledgement: None}

\section{Conflict of Interest: None}

\section{Source of Funding: None}

\section{Declaration of Patient Consent:}

Appropriate patient consent was taken prior to publication in the journal.

\section{REFERENCES}

1. Selma Feldman Witchel et.al. Congenital adrenal hyperplasia, J Pediatr Adolesc Gynecol, 2017 October; 30(5): 520-534.

2. Anne a. Gershon, judith breuer, jeffrey i. Cohen, randall j. Cohrs, michael d. Gershon, don gilden, charles grose, sophie hambleton, peter g. Kennedy, michael . Oxman, jane . Seward and koichi yamanishi et.al. Varicella zoster virus infection.Nat rev dis primers, 1: 15016. Doi:10.1038/nrdp.2015.16.

3. Jared lizzi, do Tyler hill, do Julian jakubowski, do et.al. Varicella zoster virus encephalitis, clinical practice and cases in emergency medicine, volume iii, no. 4: November 2019, 380-382.

4. Ann M et.al.Varicella-zoster virus. Arvin, clinical microbiology reviews, July 1996, p. 361-381.

5. Ozden turel, mustafa bakir, ismail gonen, nevin hatipoglu, cigdem aydogmus, emine hosaf, rengin siraneci Et.al.Children hospitalized for Varicella: complications and cost burden, value in health regional issues 2 (2013)226-230.

6. Ronald Albert Benton carey, vignesh kumar chandiraseharan, anitha jasper,tunny sebastian,chrusolitha gujjarlamudi, sowmya sathyendra, anand zachariah, asha mary abraham, and thambu david sudarsanam et.al.Varicella zoster virus infection of the central nervous system - 10 year experience from a tertiary hospital in south india.AprJun,2017;20(2)149-152.

7. Bozzola et al. Acute cerebellitis in Varicella: a ten year case series and systematic review of the literature. Italian journal of pediatrics 2014, 40:57 http://www.ijponline.net/content/40/1/57.

How to cite this article: Shaji AM, A. Priya, S Suwitha et.al. A case report on congenital adrenal hyperplasia, varicella zoster infection, varicella encephalopathy \& cerebellitis. International Journal of Science \& Healthcare Research. 2021; 6(2): 308-311. DOI: https:// doi.org/10.52403/ijshr.20210455 